\title{
PHOTOCATALYTIC DEGRADATION OF PHARMACEUTICAL DRUG ZIDOVUDINE BY UNDOPED AND 5 \% BARIUM DOPED ZINC OXIDE NANOPARTICLES DURING WATER TREATMENT: SYNTHESIS AND CHARACTERISATION
}

\section{VIJAYKUMAR S. BHAMARE*, RAVIRAJ M. KULKARNI}

Department of Chemistry, KLS Gogte Institute of Technology (Autonomous), Affiliated to Visvesvaraya Technological University Belagavi 590008, Karnataka, India

Email: vbhamare37@gmail.com

Received: 17 Oct 2018, Revised and Accepted: 12 Dec 2018

\section{ABSTRACT}

Objective: To study the photocatalytic degradation of pharmaceutical drug zidovudine (ZDV) by synthesized undoped zinc oxide nanoparticles (ZONPs) and 5\% (mole ratio) barium doped zinc oxide nanoparticles (BZONPs) during water treatment.

Methods: Kinetics studies were carried out with the help of UV-Visible Spectrophotometer. High-Resolution Mass Spectrophotometry (HR-MS) was used to identify products. A photo-reactor with mercury lamp was used as an external source of light energy. Optical power meter was used for the measurement of light intensity. The particle size of the synthesized photocatalysts was identified with the help of siemens x-ray diffractometer (XRD). The surface topography of photocatalysts was done by scanning electron microscope (SEM). Transmission electron microscopy (TEM) was used for the studies of particle size and morphology.

Results: Five degraded products of ZDV are identified by HR-MS. A suitable electron-hole pair mechanism is projected. XRD patterns show that the intensity of peak is slightly stronger in ZONPs. There is an increase in the rate of photocatalytic degradation of ZDV by adding different quantities of photocatalyst from $0.05 \mathrm{~g} \mathrm{l}^{-1}$ to $0.1 \mathrm{~g} \mathrm{l}^{-1}$. The kinetic data reveals that there is an initial increase in the values of rate constants with the increase in the concentration of ZDV. The kinetic data indicate that the values of rate constants are higher at $\mathrm{pH}=9$. There is an increase in the rate constant values with an increase in the light intensities of UV lamp.

Conclusion: The rates of photocatalytic degradation of ZDV were found to be higher using $5 \%$ (mole ratio) BZONPs as a photocatalyst.

Keywords: Water treatment, Pharmaceutical emerging contaminants, Nanotechnology, Doped zinc oxide nanoparticles, Characterisation, Photocatalysis

(C) 2019 The Authors. Published by Innovare Academic Sciences Pvt Ltd. This is an open-access article under the CC BY license (http://creativecommons.org/licenses/by/4.0/) DOI: http://dx.doi.org/10.22159/ijap.2019v11i1.30350

\section{INTRODUCTION}

There are the huge number of harmful pharmaceutical drugs detected in environmental waters that badly affects human health and animals [1]. Due to this, a systematic study is very much needed to understand the transport, occurrence, and fate of pharmaceutical drugs present in environmental waters as per world health organization (WHO) report [2]. Many oxidations techniques are reported for the elimination of harmful organic micro-contaminants found in environmental waters [3]. The complete elimination of the harmful organic microcontaminants is not feasible by conventional and biological methods [4]. Chlorination is commonly used to disinfect water by killing harmful germs and bacteria, but the by-products of this conventional method are harmful to living organisms $[5,6]$. Heptavalent Manganese (HVMG) displays disinfection property because it oxidises organic micro-contaminants present in environmental waters [7, 8]. HVMG shows chromaticity in the treated environmental waters as a major shortcoming of this oxidant [9-11].

Heterogeneous catalysis is preferred over homogeneous catalysis due to its low cost, easy recovery of catalyst and multiple active sites [12]. In view of this, there was a need of new innovative techniques for complete mineralization of harmful organic micro-contaminants [13, 14]. The constant rate values are higher in advanced oxidation processes (AOPs) than traditional biological methods which make AOPs a better option for water treatment process $[15,16]$. AOPs are found to be potent for destroying pathogens present in environmental waters [17].

Zinc oxide (ZO) has been seen as a better choice due to its special features such as low cost and absorbs the huge fraction of light quanta $[18,19]$. ZO shows its photocatalytic activity in acidic as well as basic conditions [20]. Properties of materials change due to change in size close to nanoscale [21]. Zinc oxide nanoparticles (ZONPs) have a large number of active sites which makes it potent photocatalyst for the degradation of organic substrates [22, 23].
ZONPs can be decorated by doping suitable metals to prevent the wasteful recombination of an electron-hole pair which improves the rate of photocatalytic degradation of harmful organic substrates at the surface of doped ZONPs [24, 25]. The doping will reduce the band gap energy in metal-doped ZONPs as compared to undoped ZONPs [26]. The number of surface defects is observed more in the case of doped ZONPs which increases the rate of photocatalytic degradation [27].

Antiretroviral drugs are designed to slow down the growth of viruses which cause infections in humans and animals [28]. Zidovudine (ZDV) was approved as the first antiretroviral agent for the treatment of acquired immune deficiency syndrome (AIDS) [29]. Many research studies have reported the presence of antiretroviral drugs in different aqueous systems [30, 31]. ZDV has molecular formula $\mathrm{C}_{10} \mathrm{H}_{13} \mathrm{~N}_{5} \mathrm{O}_{4}$ and molecular mass $267.24 \mathrm{~g} \mathrm{~mol}^{-1}[32,33]$. There are no reports found on photocatalytic degradation of ZDV by barium doped zinc oxide nanoparticles (BZONPs). The present study includes the effects of various parameters on photocatalytic degradation of ZDV. The photodegraded products of ZDV were identified, and a suitable mechanism is presented.<smiles>Cc1cn(C2CC(N)C(CO)O2)c(=O)[nH]c1=O</smiles>

Chemical structure of ZDV 


\section{MATERIALS AND METHODS}

\section{Chemical reagents}

Analytical grade zinc nitrate tetrahydrate, sodium hydroxide, and barium nitrate were purchased from HIMEDIA. The sample of zidovudine was received as a model compound from Sigma Aldrich. All freshly prepared solutions were used to obtain accurate results. Acetate ( $\mathrm{pH} 4.0-5.0)$, phosphate $(\mathrm{pH} \mathrm{6.0-8.0)} \mathrm{and} \mathrm{borate}(\mathrm{pH} 9.0)$ buffers were prepared.

\section{Instruments and methods}

A CARY 50 Bio UV-Vis spectrophotometer (Varian BV, The Netherlands) with the temperature controller and high-performance liquid chromatography (HPLC) (Shimadzu Prominence) were used. In order to illuminate the surface of ZONPs and BZONPs photocatalysts, a photo-reactor with a mercury lamp (PHILIPS, TUV $8 \mathrm{~W} \mathrm{~T} 5, \mathrm{E}_{\max }=254 \mathrm{~nm}$ ) as an external source of light energy was used to maintain typical light intensity. Optical power meter (Newport 2936-C) was used for the measurement of light intensity. ZONPs and BZONPs were synthesized and characterized. The particle size of synthesized ZONPs and BZONPs were identified with the help of siemens x-ray diffractometer (XRD) (Cu source) AXS D5005. The surface topography of synthesized ZONPs and BZONPs was done by scanning electron microscope (SEM) JEOL JSM 6360. Transmission electron microscopy (TEM) JEOL JEM-2010 was used for the studies of particle size and morphology of photocatalysts. Kinetic results were reproduced with the help of UV-Vis spectrophotometer (Varian BV, The Netherlands) with the temperature controller and various experiments were carried out three times by using semiconducting materials ZONPs and 5\% (mole ratio) BZONPs as a photocatalyst for the photocatalytic degradation of ZDV. Absorbance of the reaction mixtures was recorded every time within a day. The rate constants were evaluated for each experiment. Experimental data reveals that the constant rate values are almost the same for the same kind of experiment at the same temperature and suggesting good reproducibility of the obtained results. The experimentally determined rate constants were reproducible within $\pm 6 \%$. All experiments were performed in triplicates, and all points in the fig. are the mean of the results and error bars show the standard deviation of the means.

\section{Synthesis of ZONPs and BZONPs}

ZONPs and $5 \%$ (mole ratio) BZONPs were synthesized by chemical precipitation method. $100 \mathrm{ml}^{\text {zinc nitrate }}\left(0.1 \mathrm{~mol} \mathrm{l}^{-1}\right)$ and $100 \mathrm{ml}$ sodium hydroxide $\left(0.2 \mathrm{~mol} \mathrm{l}^{-1}\right)$ aqueous solutions were prepared in double distilled water. Now, $100 \mathrm{ml}$ zinc nitrate solution was taken in a beaker and placed on a magnetic stirrer. In order to inhibit the anomalous growth of zinc hydroxide crystals, $10 \mathrm{mg} \mathrm{l}^{-1}$ of sodium dodecyl sulphate was mixed as a surfactant into the beaker [34]. This reaction mixture was kept for continuous stirring for the period of $2 \mathrm{~h}$. Then, $100 \mathrm{ml}$ of $0.2 \mathrm{~mol} \mathrm{l}^{-1}$ sodium hydroxide solution was added drop by drop with continuous stirring (2000 rpm) to form the crystals of zinc hydroxide. After stirring for the period of $5 \mathrm{~h}$, the reaction mixture was kept undisturbed for $12 \mathrm{~h}$ to discard the supernant part of the reaction mixture. The white precipitate was then centrifuged. After this, the white precipitate was washed with the help of double distilled water and subsequently with ethanol many times in order to remove impurities. This zinc hydroxide precipitate was dried at temperature $110^{\circ} \mathrm{C}$ in an oven for $5 \mathrm{~h}$ and kept undisturbed for the period of at least $1 \mathrm{~h}$ to maintain the room temperature. This precipitate was transferred into the mortar and grounded into powder form. Then, the white powder was transferred into a crucible for the calcination process at $500{ }^{\circ} \mathrm{C}$ for the period of $2 \mathrm{~h}$ in a muffle furnace to convert it into ZONPs. Similarly, 5\% (mole ratio) BZONPs were also synthesized. In order to prepare $5 \%$ (mole ratio) BZONPs, $0.05 \mathrm{~mol} \mathrm{l}^{-1} \mathrm{Ba}\left(\mathrm{NO}_{3}\right)_{2}$ solution was added into the reaction mixture containing $0.1 \mathrm{~mol} \mathrm{l}^{-1}$ zinc nitrate solution and $10 \mathrm{mg} \mathrm{l}^{-1}$ of sodium dodecyl sulphate as a surfactant. Sodium hydroxide solution $\left(0.2 \mathrm{~mol} \mathrm{l}^{-1}\right)$ is added drop by drop with constant magnetic stirring. The same procedure is repeated to enter the barium into interstitial positions of ZONPs lattice structure [35].

\section{The photocatalytic degradation process}

A suitable quantity of ZDV solution was poured into the Pyrex beaker containing a suitable buffer solution $\left(0.2 \mathrm{~mol} \mathrm{l}^{-1}\right)$. In this reaction mixture, ZONPs were added and kept undisturbed for a period of $2 \mathrm{~h}$ in a dark room to attain the equilibrium of adsorption and desorption between photocatalyst and organic substrate. Then, the reaction mixture was kept into the photo-reactor for illumination of the surface of ZONPs with the help of Philips $8 \mathrm{~W}$ UV lamps at $254 \mathrm{~nm}$. This reaction mixture was kept for continuous stirring inside the photo-reactor. Then, the degraded solution was taken out and kept for the process of centrifugation at $5000 \mathrm{rpm}$ for $10 \mathrm{~min}$. Then, the reaction mixture was taken into a cuvette for the measurement of absorbance at $267 \mathrm{~nm}$. This kinetic study was performed to examine the degree of mineralization of organic substrate ZDV by ZONPs. Similarly, the above photocatalytic degradation process was repeated for $5 \%$ (mole ratio) BZONPs. Lambert-Beers law was verified for $[\mathrm{ZDV}]=1.0 \times 10^{-6}$ to $1.0 \times 10^{-5}$ mol $\mathrm{l}^{-1}$ at $267 \mathrm{~nm}$.

\section{RESULTS AND DISCUSSION}

Comparison of different photocatalysts for the degradation of ZDV

Rate constant values for the mineralization of ZDV by UV/5\% (mole ratio) BZONPs were found to be higher as compare to UV and UV/ZONPs treatments as shown in fig. 1 and presented in table 1 .

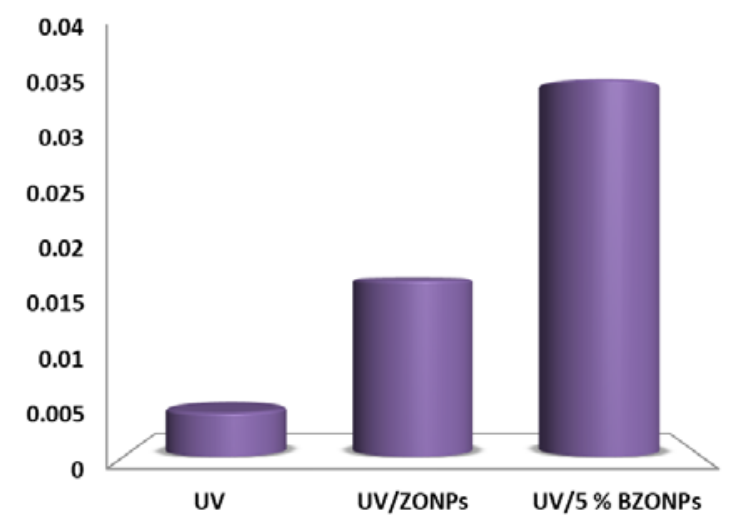

Fig. 1: Rate constants for the photocatalytic degradation of ZDV by various treatments

Table 1: Rate constant values for the photo catalytic degradation of ZDV by UV, UV/ZONPs and UV/5\% (mole ratio) BZONPs photocatalysts at $[\mathrm{ZDV}]=1 \times 10^{-5} \mathrm{~mol} \mathrm{l}^{-1}$ and $\mathrm{pH}=8$

\begin{tabular}{ll}
\hline Different treatments & Rate constants $\left.\mathbf{k}_{\text {obs }} \mathbf{x} \mathbf{1 0}^{\mathbf{3}} \mathbf{( m i n}^{-1}\right)$ \\
\hline UV & 4.26 \\
UV/ZONPs & 16.91 \\
UV/5\% (mole ratio) BZONPs & 36.12 \\
\hline
\end{tabular}

This confirms that water treatment by $5 \%$ (mole ratio) BZONPs photocatalyst is more effective than UV and UV/ZONPs treatments for the photocatalytic degradation of ZDV. The influence of barium doping on the photocatalytic degradation of ZDV was investigated thoroughly by using $5 \%$ (mole ratio) BZONPs because $5 \%$ doping of barium into the $\mathrm{ZnO}$ lattice reduces the particles size and increases 
the surface reactivity of the photocatalyst which slows down the rate of recombination of photo-generated electron and hole [36]. It means that $5 \%$ (mole ratio) BZONPs increases the surface charge transfer for the higher degree of mineralization of ZDV. Therefore, the detail investigation was performed by using the $5 \%$ (mole ratio) BZONPs.

\section{Characterization of ZONPs and 5\% (mole ratio) BZONPs}

\section{XRD and scherrer equation}

XRD was used to find out the particle size and phase purity of ZONPs and $5 \%$ (mole ratio) BZONPs. The X-ray diffraction was performed at 2 degrees per min to get the binary output. The XRD pattern shows intense sharp diffraction peaks at the reflection from (100), (002), (101), (102), (110), (103), (200) and (112) crystal planes of the hexagonal wurzite structure of ZONPs and 5\% (mole ratio) BZONPs. No other additional peak related to secondary phases of ZONPs and $5 \%$ (mole ratio) BZONPs were observed in the XRD pattern. This also confirms the proper doping of barium metal into the interstitial position of ZONPs lattice. This observation indicates that the hexagonal wurzite structure of ZONPs is not disturbed by decorating it with $5 \%$ (mole ratio) barium metal. This confirms that the prepared ZONPs and $5 \%$ (mole ratio) BZONPs are found to be in a single phase. There is a sharp, intense diffraction peak observed at the crystal plane (101) in XRD pattern of both ZONPs and $5 \%$ (mole ratio) BZONPs. This observation indicates that ZONPs and $5 \%$ (mole ratio) BZONPs growth might have taken place along the easy direction of crystallization [37]. XRD patterns of ZONPs and $5 \%$ (mole ratio) BZONPs indicates that the intensity of peak is slightly stronger in ZONPs. The particle sizes of photocatalysts were calculated by Scherrer Eqn (1) [38].

$$
D=k \lambda / \beta \cos \theta
$$

In this Scherrer Eqn, 0 denotes X-ray wavelength in angstrom, $\boldsymbol{\theta}$ denotes Bragg diffraction angle, $\mathrm{D}$ denotes average crystalline diameter, $\boldsymbol{\beta}$ is the full width at half-maximum (WDHM) and $\boldsymbol{k}$ is the dimensionless shape factor $(0.94 \lambda)$. The X-ray wavelength for the XRD was $0.154 \times 10^{-9} \mathrm{~m}$. The broadening of the sharp intense peak observed at crystal plane (101) was made to calculate the particle size of the ZONPs and $5 \%$ (mole ratio) BZONPs by Scherrer Eqn (1). After putting the values of $\boldsymbol{\lambda}, \boldsymbol{k}$ and $\boldsymbol{\beta}$ in the Scherrer Eqn (1), calculated values of average grain size for ZONPs is $35.08( \pm 3) \mathrm{nm}$ and $5 \%$ (mole ratio) BZONPs is $24.39( \pm 2) \mathrm{nm}$. The higher value of average grain size of ZONPs over $5 \%$ (mole ratio) BZONPs indicate that the surface area is increased in the case of $5 \%$ (mole ratio) BZONPs and hence the rate of photocatalytic degradation of ZDV by $5 \%$ (mole ratio) BZONPs photocatalyst is faster. The XRD pattern of ZONPs and 5\% (mole ratio) BZONPs are presented in fig. 2.

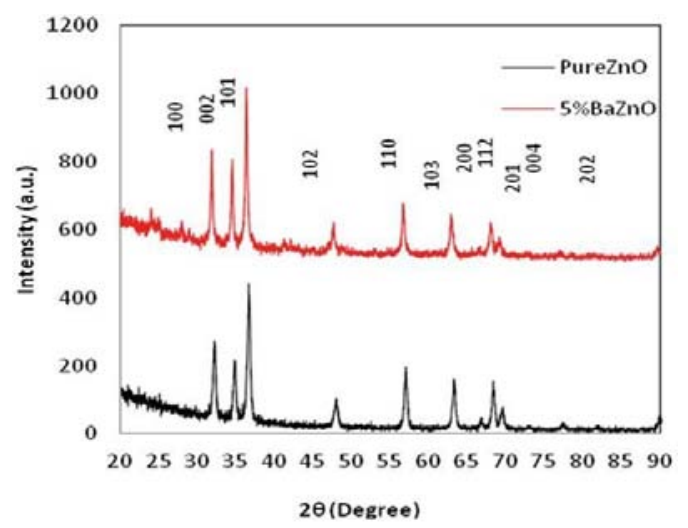

Fig. 2: XRD patterns of ZONPs and 5\% (mole ratio) BZONPs photocatalysts

\section{SEM of ZONPs and 5\% (mole ratio) BZONPs photocatalysts}

The surface morphology was studied using SEM. The size, shape, and morphologies of ZONPs and $5 \%$ (mole ratio) BZONPs were analyzed from SEM images. A voltage of $20 \mathrm{kv}$ was supplied, and the microscope was adjusted for obtaining large images of photocatalysts. The precision distance was kept $1 \mu \mathrm{m}$ length. The particles can be seen in the SEM images of ZONPs and $5 \%$ (mole ratio) BZONPs. These SEM images show that both ZONPs and $5 \%$ (mole ratio) BZONPs are uniform and homogeneous with closely aggregate oval in shape. The size of particles of $5 \%$ (mole ratio) BZONPs was found to be slightly bigger than ZONPs. This observation confirms that there is an increase in the surface area of $5 \%$ (mole ratio) BZONPs which increases the rate of photocatalytic degradation of ZDV into harmless substances. SEM images are shown in fig. 3a and fig. $3 b$.

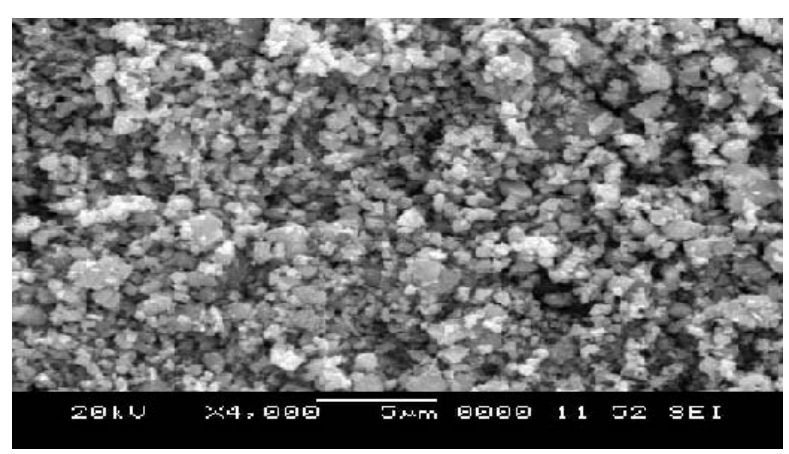

Fig. 3a: SEM Micrographs of ZONPs

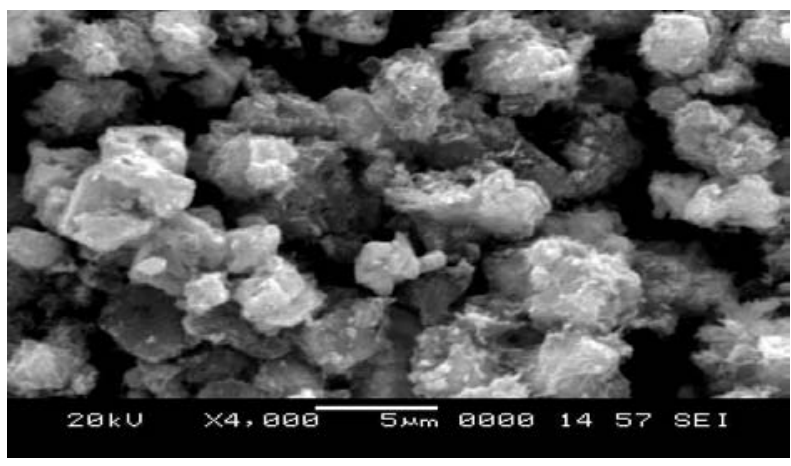

Fig. 3b: SEM micrographs of $5 \%$ (mole ratio) BZONPs

\section{EDX of ZONPs and 5\% (mole ratio) BZONPs photocatalysts}

The quantitative elemental analysis for synthesized ZONPs and 5\% (mole ratio) BZONPs were carried out by EDX (JEOL JED-2300). The results of EDX shows that there are only three elements present in 5 $\%$ (mole ratio) BZONPs and no any other element is found as an impurity in this pattern. EDX of $5 \%$ (mole ratio) BZONPs shows that the mass percentage of zinc is 71.2 , oxygen is 23.9 and barium is 4.78. The atomic percentages of zinc, oxygen and barium elements obtained from EDX of $5 \%$ (mole ratio) BZONPs were found to be 41.6, 57.1 and 1.33 respectively. EDX for ZONPs and 5\% (mole ratio) BZONPs is presented in fig. $4 \mathrm{a}$ and fig. $4 \mathrm{~b}$ respectively.

\section{TEM of ZONPs and 5\% (mole ratio) BZONPs photocatalysts}

TEM (JEOL JEM-2010) was used to do an analysis of particle size and morphology of ZONPs and $5 \%$ (mole ratio) BZONPs. TEM micrograph of $5 \%$ (mole ratio) BZONPs shows heterogeneously dispersed aggregates having a cylindrical shape and crystalline structures. Dispersion of small dark spots was observed in TEM micrograph of $5 \%$ (mole ratio) BZONPs which indicates doping of 5 $\%$ (mole ratio) barium metal into interstitial sites of the lattice. $5 \%$ (mole ratio) BZONPs are having breadth from 15-20 nm and length from 25-30 nm approximately. The crystallite size of prepared nanoparticles is close to ZONPs data received from XRD values. TEM micrographs of ZONPs and $5 \%$ (mole ratio) BZONPs are shown in fig. $5 \mathrm{a}$ and fig. $5 \mathrm{~b}$. 


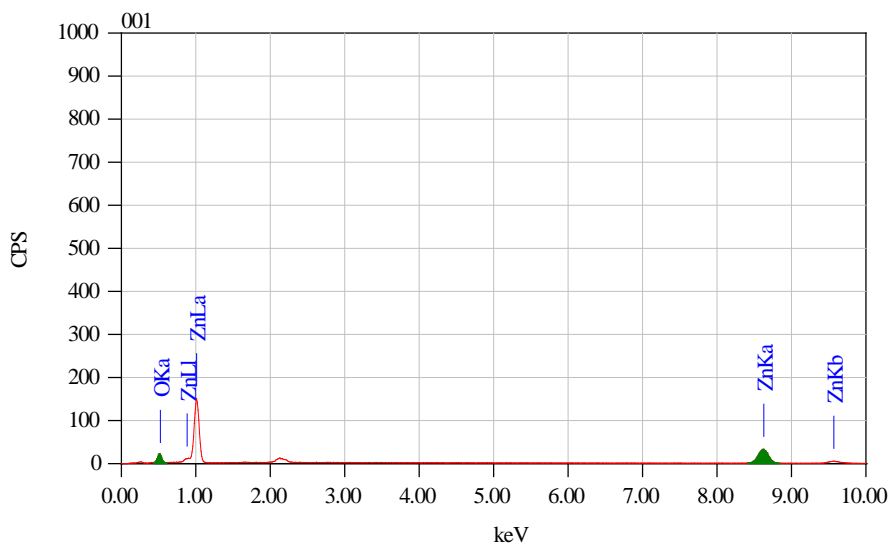

Fig. 4a: EDX analysis of semiconducting material pure ZONPs

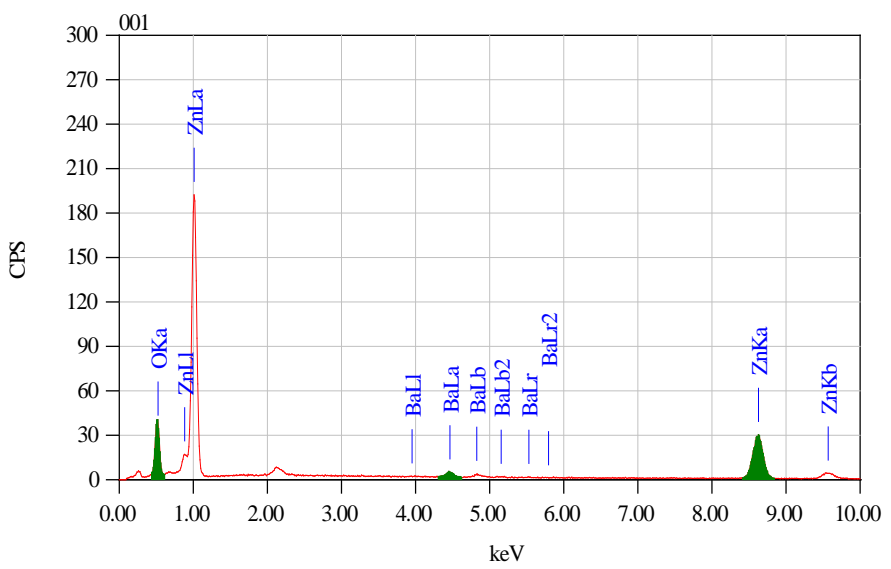

Fig. 4b: EDX analysis of 5\% (mole ratio) BZONPs

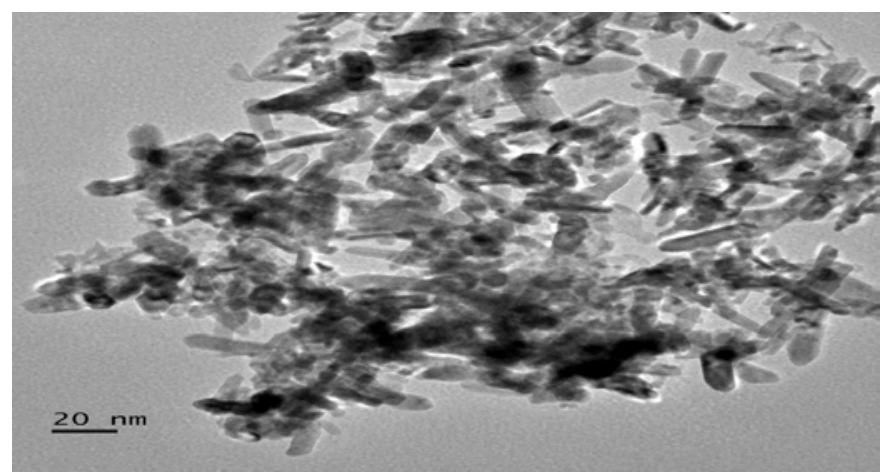

Fig. 5a: TEM micrographs of ZONPs

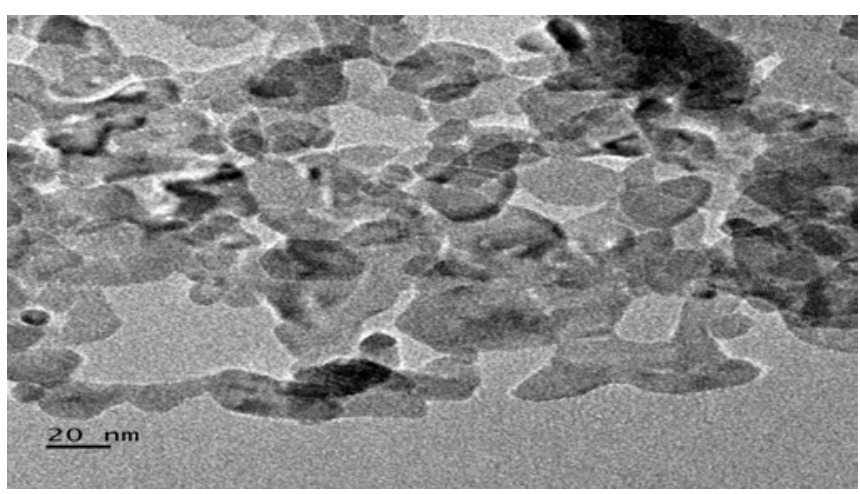

Fig. 5b: TEM micrographs of 5\% (mole ratio) BZONPs 
Influence of loading of photocatalyst on the rate of photocatalytic degradation reactions

The influence of ZONPs and $5 \%$ (mole ratio) BZONPs on the photodegradation of ZDV was studied by adding different quantities of photocatalyst varying from $0.05 \mathrm{~g} \mathrm{l}^{-1}$ to $0.25 \mathrm{~g} \mathrm{l}^{-1}$ into the reaction mixture and placed it in a photo-reactor at fixed $[\mathrm{ZDV}]=1 \times 10^{-5} \mathrm{~mol} \mathrm{l}^{-1}$ and $\mathrm{pH}=8$. The experimental data shows that there is an increase in the rate of photocatalytic degradation from $0.05 \mathrm{~g} \mathrm{l}^{-1}$ to $0.1 \mathrm{~g} \mathrm{l}^{-1}$ but after that, the rate almost remains constant as presented in fig. 6 and listed in table 2 for ZONPs and $5 \%$ (mole ratio) BZONPs.

Table 2: Influence of loading of different quantities of photocatalyst ZONPs and $5 \%$ (mole ratio) BZONPs separately at [ZDV] $=1 \mathrm{x10}^{-5} \mathrm{~mol}^{-1}$

\begin{tabular}{|c|c|c|}
\hline ZONPs or $5 \%$ (mole ratio) [BZONPs] $\mathrm{g} \mathrm{l}^{-1}$ & $\begin{array}{l}k_{\text {obs }} \times 10^{3}\left(\mathrm{~min}^{-1}\right) \\
\text { with ZONPs }\end{array}$ & $\begin{array}{l}k_{\text {obs }} \times 10^{2}\left(\min ^{-1}\right) \\
\text { with } 5 \% \text { (mole ratio) BZONPs }\end{array}$ \\
\hline 0.025 & 9.00 & 1.80 \\
\hline 0.050 & 12.30 & 2.46 \\
\hline 0.100 & 18.20 & 3.64 \\
\hline 0.200 & 13.70 & 2.74 \\
\hline 0.250 & 10.80 & 2.16 \\
\hline
\end{tabular}

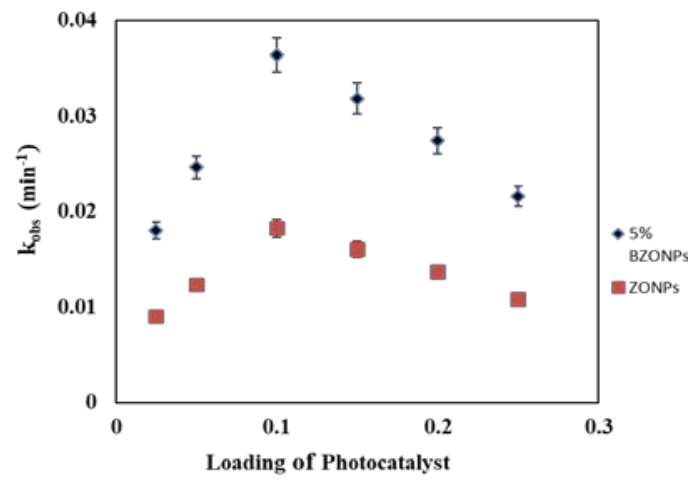

Fig. 6: Influence of loading of different quantities of photocatalyst ZONPs and $5 \%$ (mole ratio) BZONPs separately at $[\mathrm{ZDV}]=1 \times 10^{-5} \mathrm{~mol} \mathrm{l}^{-1}$

The further addition of ZONPs and $5 \%$ (mole ratio) BZONPs into the reaction mixtures decreases the constant rate values. The decrease in observed rate constant $\left(\mathrm{k}_{\mathrm{obs}}\right)$ values after the limiting value of 0.1 $\mathrm{g} \mathrm{l}^{-1}$ may be due to the increase in turbidity of the reaction mixture which prevents UV radiations from reaching to the surface of the photocatalyst and slowing down the speed of photocatalytic degradation. The initial increase in the rate constant values of the photocatalytic degradation may be due to increase in surface area and more number of active sites of ZONPs and $5 \%$ (mole ratio) BZONPs which may increase the hydroxyl species to degrade more number of substrate molecules adsorbed on the surface of photocatalyst [39].

\section{Effect of change in [ZDV]}

The [ZDV] was varied in the rage of $2 \times 10^{-6}$ to $2 \times 10^{-5} \mathrm{~mol} \mathrm{l}^{-1}$ and maintaining other reaction conditions fixed such as the concentration of $5 \%$ (mole ratio) BZONPs $=1 \mathrm{~g} \mathrm{l}^{-1}$ and $\mathrm{pH}=8$. The kinetic data reveals that there is an initial increase in the values of $\mathrm{k}_{\mathrm{obs}}$ with an increase in [ZDV]. The reason behind it may be excitation of a higher number of ZDV substrate molecules which are then adsorbed on the surface of $5 \%$ (mole ratio) BZONPs. It is also interesting to note that $\mathrm{k}_{\mathrm{obs}}$ values reach to a maximum value at the higher concentration of $1 \times 10^{-5} \mathrm{~mol} \mathrm{l}^{-1}$ of ZDV. Then, there is decline in the values of kobs as shown in fig. 7 and listed in table 3 for UV, $\mathrm{UV} /$ ZONPs and UV/5\% (mole ratio) BZONPs treatments. The decline in the $\mathrm{k}_{\mathrm{obs}}$ values beyond the higher concentration of $1 \times 10^{-5} \mathrm{~mol} \mathrm{l}^{-1}$ of ZDV may be due to the excess number of substrate molecules which acts as a filter for the UV radiations and thereby responsible for the decrease in the degree of mineralization of substrate [40].

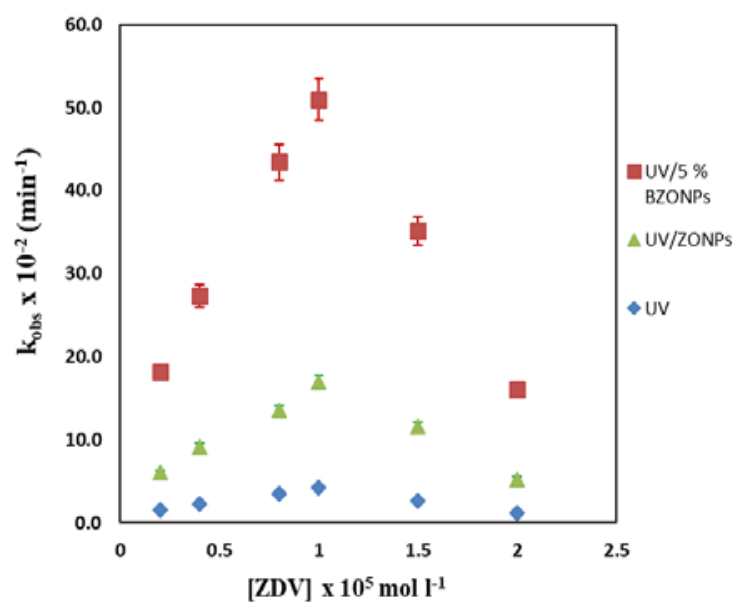

Fig. 7: Effect of varying [ZDV] from $2 \times 10^{-6}$ to $2 \times 10^{-5} \mathrm{~mol} \mathrm{l}^{-1}$ on the rate of photodegradation of ZDV by UV, UV/ZONPs and UV/5 $\%$ (mole ratio) BZONPs at $\mathrm{pH}=8$

Table 3: Effect of varying [ZDV] from $2 \times 10^{-6}$ to $2 \times 10^{-5} \mathrm{~mol}^{-1}$ on the rate of photo degradation of ZDV by UV, UV/ZONPs and UV/5 \% (mole ratio) BZONPs at $\mathrm{pH}=8$

\begin{tabular}{|c|c|c|c|}
\hline$[\mathrm{ZDV}] \times 10^{5} \mathrm{~mol} \mathrm{l}^{-1}$ & $\begin{array}{l}k_{\text {obs }} \times 10^{3}\left(\min ^{-1}\right) \\
\text { with } U V\end{array}$ & $\begin{array}{l}k_{\text {obs }} \times 10^{3}\left(\min ^{-1}\right) \\
\text { with ZONPs }\end{array}$ & $\begin{array}{l}\mathbf{k}_{\text {obs }} \times 10^{2}\left(\mathrm{~min}^{-1}\right) \text { with } \\
5 \% \text { (mole ratio) BZONPs }\end{array}$ \\
\hline 0.2 & 1.51 & 6.04 & 0.68 \\
\hline 0.4 & 2.27 & 9.11 & 1.42 \\
\hline 0.8 & 3.47 & 13.48 & 2.91 \\
\hline 1.0 & 4.24 & 16.91 & 3.61 \\
\hline 1.5 & 2.62 & 11.56 & 3.51 \\
\hline 2.0 & 1.13 & 5.24 & 0.65 \\
\hline
\end{tabular}

Effect of varying $\mathrm{pH}$ of reaction mixtures of ZDV and $5 \%$ (mole ratio) BZONPs

The $\mathrm{pH}$ was varied in the rage of 5.0 to 9.0 to study its effect on the photocatalytic degradation of ZDV by maintaining other reaction conditions constants. The absorbance of the reaction mixture was recorded from $\mathrm{pH} 5.0$ to 9.0 with the help of UV-Vis spectrophotometer. The graph of $\mathrm{k}_{\text {obs }}\left(\mathrm{min}^{-1}\right)$ versus $\mathrm{pH}$ was plotted as presented in fig. 8 and table 4 . The kinetic data indicates that the values of rate constants are lower at the $\mathrm{pH}=5$ and higher at the $\mathrm{pH}=9$. 
Table 4: Effect of varying $\mathrm{pH}$ on the rate of photo degradation of ZDV by using $5 \%$ (mole ratio) BZONPs, [5\% BZONPs] $=1 \mathrm{~g} \mathrm{l}^{-1}$ and [ZDV] $=1 \times 10^{-5} \mathrm{~mol} \mathrm{l}^{-1}$

\begin{tabular}{ll}
\hline $\mathbf{p H}$ & $\mathbf{k}_{\text {obs }} \mathbf{x} \mathbf{1 0}^{\mathbf{2}}\left(\mathbf{m i n}^{-1}\right)$ \\
\hline 4.0 & 1.90 \\
7.0 & 2.89 \\
9.0 & 3.65 \\
\hline
\end{tabular}

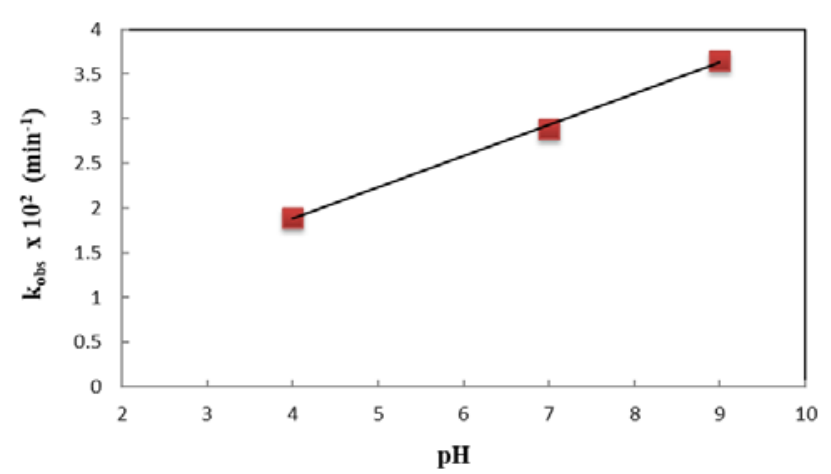

Fig. 8: Effect of varying $\mathrm{pH}$ on the rate of photo degradation kinetics of ZDV by using $5 \%$ (mole ratio) BZONPs, [5\% BZONPs] $=1 \mathrm{~g}^{-1}$ and $[\mathrm{ZDV}]=1 \times 10^{-5} \mathrm{~mol} \mathrm{l}^{\mathrm{l}}$

The reason behind this may be increase in more number of hydroxide ions at $\mathrm{pH}=9$ which are responsible for the generation of more number of powerful hydroxyl radicals by combining with holes. As a result of this the photocatalytic rate degradation at $\mathrm{pH}=9$ is higher. It means that at higher alkaline $\mathrm{pH}$, smaller particle size and more crystallanity of 5 (mole ratio) \% BZONPs shows the faster degree of mineralization of organic substrate ZDV with the help of source of light energy.

\section{Influence of light intensity on the photodegradation of organic} substrate ZDV

The influence of UV light intensity on the values of $\mathrm{k}_{\mathrm{obs}}\left(\mathrm{min}^{-1}\right)$ was investigated by changing the distance of UV lamp from the reaction mixture. The kinetic data implies that the $\mathrm{k}_{\mathrm{obs}}$ values are found higher at the higher light intensities of UV lamp for the degree of mineralization of organic substrate ZDV present in environmental waters. The reason behind this increase in the kobs values may be due to increase in the number of photons bombarding per unit area of $5 \%$ (mole ratio) BZONPs. As a result of this, there is an increase in the number of photo-generated electron-hole pairs. The large number of photo-generated holes degrades the organic substrate ZDV adsorbed on $5 \%$ (mole ratio) BZONPs and forms simple substances during the water treatment process [41]. The graph $\mathrm{k}_{\mathrm{obs}}$ $\left(\mathrm{min}^{-1}\right)$ versus variation in the intensity of UV lamp was plotted and presented in fig. 9. The values of $\mathrm{k}_{\mathrm{obs}}\left(\mathrm{min}^{-1}\right)$ are listed in table 5 for the photodegradation of ZDV. Thus, the variation in the intensity of the UV lamp increases the photocatalytic activity of $5 \%$ (mole ratio) BZONPs.

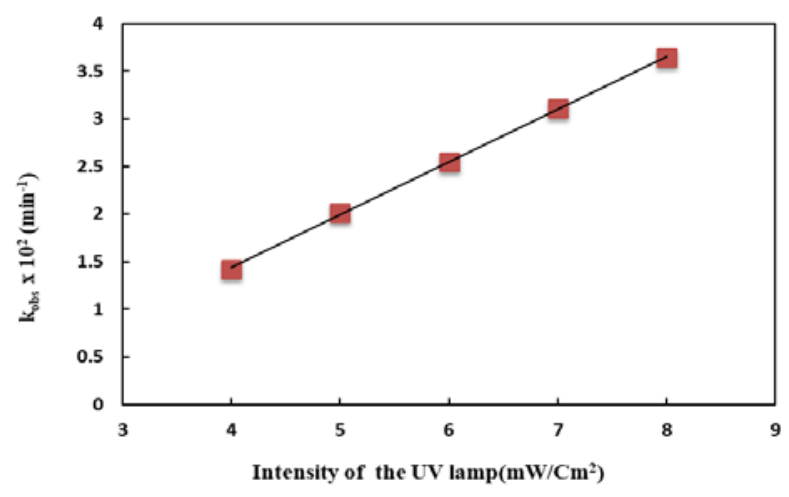

Fig. 9: Influence of variation in light intensity on photo degradation of ZDV by $5 \%$ (mole ratio) BZONPs, [5\% BZONPs] = $1 \mathrm{~g} \mathrm{l}^{-1},[\mathrm{ZDV}]=1 \times 10^{-5} \mathrm{~mol} \mathrm{l}^{-1}$ and $\mathrm{pH}=8$

Table 5: Influence of variation in light intensity on photo degradation of ZDV by $5 \%$ (mole ratio) BZONPs, [5\% BZONPs] = $1 \mathrm{~g} \mathrm{l-1}$, [ZDV] = 1 $\times 10^{-5} \mathrm{~mol} \mathrm{l}^{-1}$ and $\mathrm{pH}=8$

\begin{tabular}{ll}
\hline Intensity of UV lamp $\mathbf{m W} / \mathbf{C m}^{2}$ & $\mathbf{k}_{\text {obs } \mathbf{x} \mathbf{1 0}^{2}\left(\mathbf{m i n}^{-1}\right)}$ \\
\hline 4.0 & 1.42 \\
5.0 & 2.01 \\
6.0 & 2.55 \\
7.0 & 3.11 \\
8.0 & 3.64 \\
\hline
\end{tabular}

Photo-generated electron-hole pair mechanism for the photocatalytic degradation of ZDV

A suitable quantity of Propan-2-ol as a scavenger of reactive species hydroxyl radicals or KI as a quencher of $\mathrm{h}^{+}$and hydroxyl radical were mixed into the reaction mixture to check the photocatalytic degradation mechanism of substrate ZDV and 5\% (mole ratio) BZONPs. The experimental results indicate that $\mathrm{h}^{+}$are reactive species in this plausible mechanism. Therefore, it is supposed that after the adsorption of ZDV on $5 \%$ (mole ratio) BZONPs at dark, the destruction of ZDV in the presence of UV light could be come first through direct reactions of ZDV with $\mathrm{h}^{+}$trapped on the photocatalyst surface.

When irradiated with sufficient wavelength of light energy, electronhole pairs are created at the surface of the $5 \%$ (mole ratio) BZONPs [42]. Most of these electron-hole pairs are recombined, but this kind of recombination of electron and hole is a wasteful process while remaining electron-hole pairs which are not recombined starts initiating redox reactions in ZDV for its total photodegradation into simple substances due to higher redox potentials of photogenerated excitons [14].

$$
\text { BZONPs }+h v \rightarrow \text { BZONPs }\left(\mathrm{h}^{+}+\mathrm{e}^{-}\right)
$$

These photo-generated holes (BZONPs) $\mathrm{h}^{+}$of the valence band (VB) interacts with $\mathrm{H}_{2} \mathrm{O}$ and generates reactive powerful hydroxyl free radical species.

$$
\text { (BZONPs) } \mathrm{h}^{+}+\mathrm{H}_{2} \mathrm{O} \rightarrow \mathrm{H}^{+}+\mathrm{OH}
$$

(BZONPs) $\mathrm{h}^{+}$may interact with hydroxyl anion to generate the reactive hydroxyl species at the VB.

$$
\text { (BZONPs) } \mathrm{h}^{+}+\mathrm{OH}^{-} \rightarrow \text {. OH }
$$


The reactive hydroxyl species at the VB of $5 \%$ (mole ratio) BZONPs interacts with the adsorbed substrate ZDV to degrade it into smaller simple substances.

$$
\text { ZDV+. } \mathrm{OH} \rightarrow \text { Photodegraded Products }
$$

Now, superoxide radical anions are generated at the conduction band (CB) due to the combination of BZONPs ( $\mathrm{e}^{-}$) with $\mathrm{O}_{2}$ molecule. This may take place due to the transfer of trapped e-from the surface of bariumto oxygen atom [43]. It avoids the wasteful recombination of photo-generated e-and $\mathrm{h}^{+}$which increases the rate of the photocatalytic degradation of ZDV molecules adsorbed at the surface of $5 \%$ (mole ratio) BZONPs.

$$
\text { (BZONPs) } \mathrm{e}^{-}+\mathrm{O}_{2} \rightarrow . \mathrm{O}^{-}{ }_{2}
$$

After this,. $\mathrm{O}_{2}^{-}$species interacts with $\mathrm{H}^{+}$ions to generate $\mathrm{HO}_{2}$. free radicals.

$$
\cdot \mathrm{O}^{-}{ }_{2+} \mathrm{H}^{+} \rightarrow \mathrm{HO}_{2} \text {. }
$$

Then, $\mathrm{HO}_{2}$. free radicals may interact with photo-generated (BZONPs)e-to form $\mathrm{HO}_{2}$-anion $[44,45]$.

$$
\mathrm{HO}_{2}+\mathrm{e}^{-} \rightarrow \mathrm{HO}_{2}
$$

$\mathrm{HO}_{2}$-anion then combines with $\mathrm{H}^{+}$ions to produce hydrogen peroxide.

$$
\mathrm{HO}_{2}{ }^{-}+\mathrm{H}^{+} \rightarrow \mathrm{H}_{2} \mathrm{O}_{2}
$$

$\mathrm{H}_{2} \mathrm{O}_{2}$ then interacts with (BZONPs) e-at the $\mathrm{CB}$ to generate-OH and. $\mathrm{OH}$ species.

$$
\mathrm{H}_{2} \mathrm{O}_{2+} \text { (BZONPs) } \mathrm{e}^{-} \rightarrow-\mathrm{OH}+. \mathrm{OH}
$$

Now,. OH species may remove a hydrogen atom from the substrate ZDV adsorbed at the surface of $5 \%$ (mole ratio) BZONPs to form photodegraded products [46-48].

$$
\cdot \mathrm{OH}+\mathrm{ZDV} \rightarrow \text { Photodegraded products }
$$

The reactions as shown in this mechanism are possible because of the presence of dissolved $\mathrm{O}_{2}$ and $\mathrm{H}_{2} \mathrm{O}$ molecules. In the absence of $\mathrm{H}_{2} \mathrm{O}$ molecules, the formation of powerful hydroxyl free radical species is not possible, and it would have been inhibited photodegradation of ZDV. The structures of various photodegraded products of ZDV labelled as ZDV_P1, ZDV_P2, and ZDV_P3 are shown in fig. 10. The UV degraded products of ZDV were identified in the range of $100-800 \mathrm{~m} / z$ and listed in table 6 .

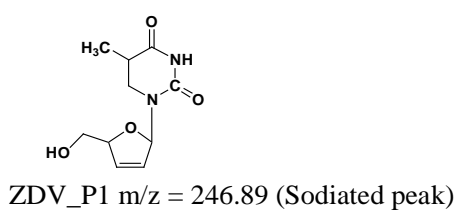

1-(5-Hydroxymethyl-2,5-dihydro-furan-2-yl)-5-methyl-dihydro-pyrimidine-2,4-dione

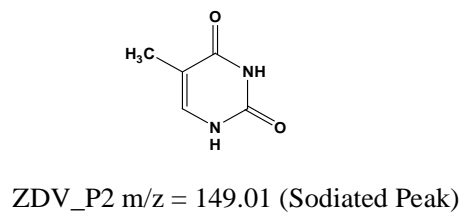

5-Methyl-1H-pyrimidine-2,4-dione

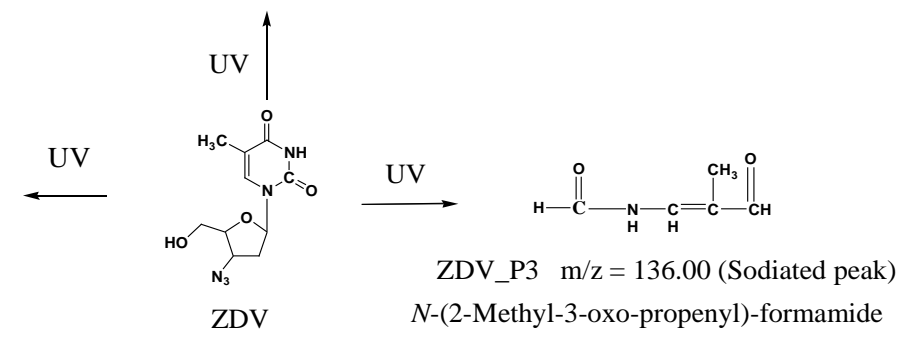

$\mathrm{m} / \mathrm{z}=290.08$ (Sodiated peak)

1-(4-Azido-5-hydroxymethyl-tetrahydro-furan-2-yl)-5-methyl-1H-pyrimidine-2,4-dione

Fig. 10: Photodegraded products of substrate ZDV by UV source of radiation 


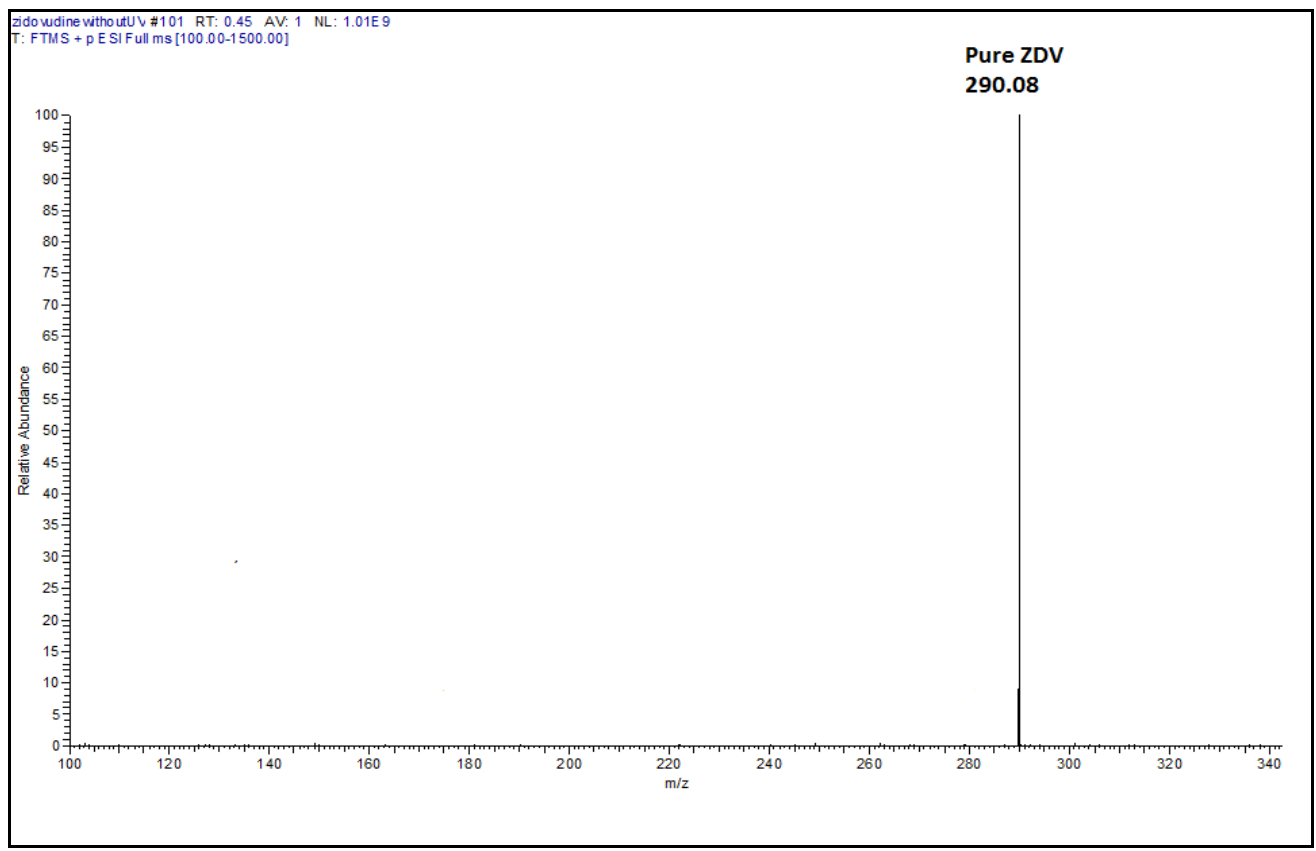

Fig. 11a: HR-MS spectra of pure ZDV

Table 6: Photocatalytic degraded products of ZDV using HR-MS

\begin{tabular}{lllll}
\hline $\begin{array}{l}\text { ZDV } \\
\text { Products }\end{array}$ & $\begin{array}{l}\text { Measured } \\
\mathbf{M}+\mathbf{H}^{+} / \mathbf{M}^{+} \mathbf{N a}^{+}\end{array}$ & $\begin{array}{l}\text { Theoretical } \\
\text { mass (Da) }\end{array}$ & $\begin{array}{l}\text { Molecular } \\
\text { formula }\end{array}$ & $\begin{array}{l}\text { Name of the identified photodegraded products of substrate } \\
\text { ZDV }\end{array}$ \\
\hline ZDV_P1 & 246.89 & 224.21 & $\mathrm{C}_{10} \mathrm{H}_{12} \mathrm{~N}_{2} \mathrm{O}_{4}$ & $\begin{array}{l}\text { 1-(5-Hydroxymethyl-2,5-dihydro-furan-2-yl)-5-methyl-1H- } \\
\text { pyrimidine-2,4-dione }\end{array}$ \\
& & & & 5-Methyl-1H-pyrimidine-2,4-dione \\
ZDV_P2 & 149.01 & 126.11 & $\mathrm{C}_{5} \mathrm{H}_{6} \mathrm{~N}_{2} \mathrm{O}_{2}$ & $\mathrm{~N}$-(2-Methyl-3-oxo-propenyl)-formamide \\
ZDV_P3 & 136.00 & 113.11 & $\mathrm{C}_{5} \mathrm{H}_{7} \mathrm{NO}_{2}$ & \\
\hline
\end{tabular}

Positive mode electrospray ionization (ESI+) technique was used for the product analysis by HR-MS. The mass spectra for pure ZDV and UV degraded ZDV are shown in fig. 11a and 11b.

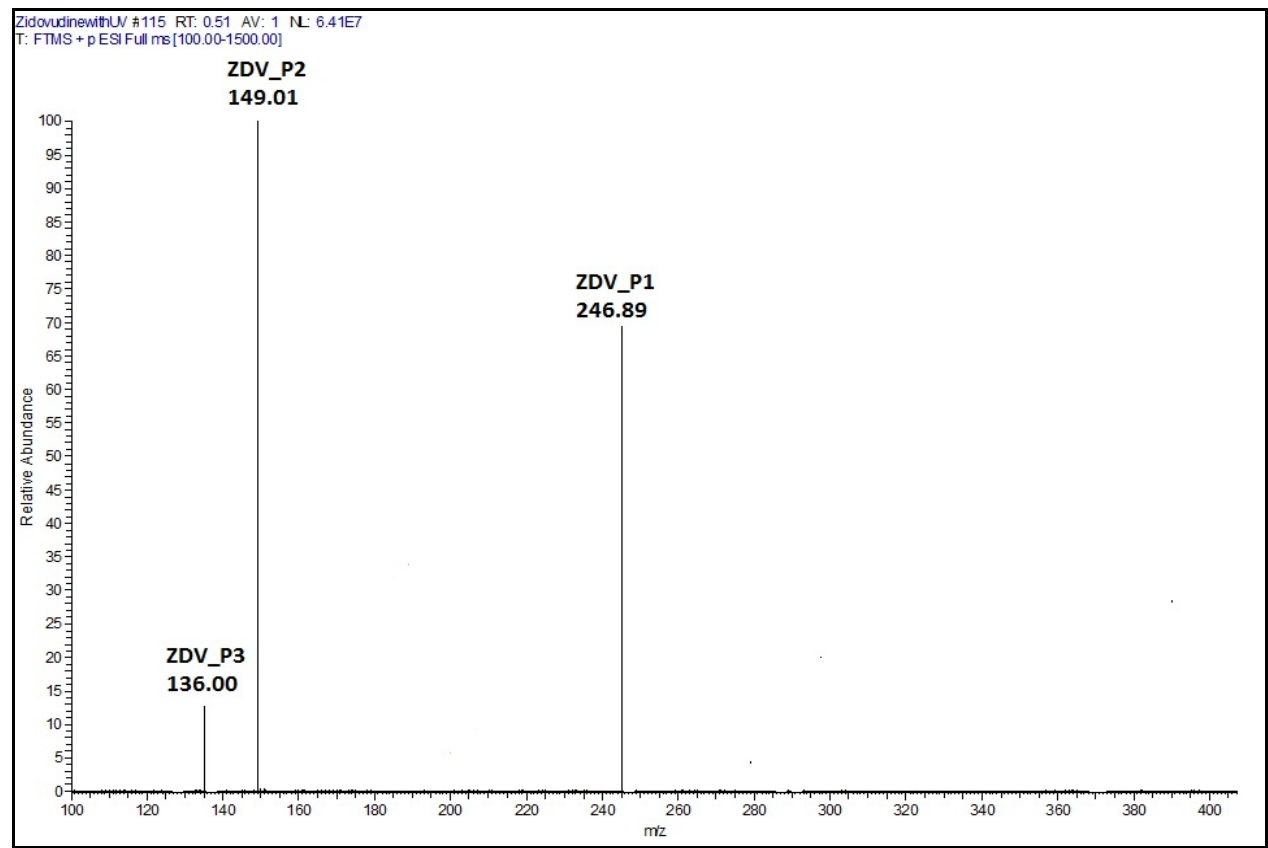

Fig. 11b: HR-MS spectra of photodegraded products of substrate ZDV, with the help of HR-MS and experimental results, the plausible photo-generated electron-hole pair photocatalytic degradation mechanism for ZDV by UV/5 \% (mole ratio) BZONPs is presented in fig. 12 


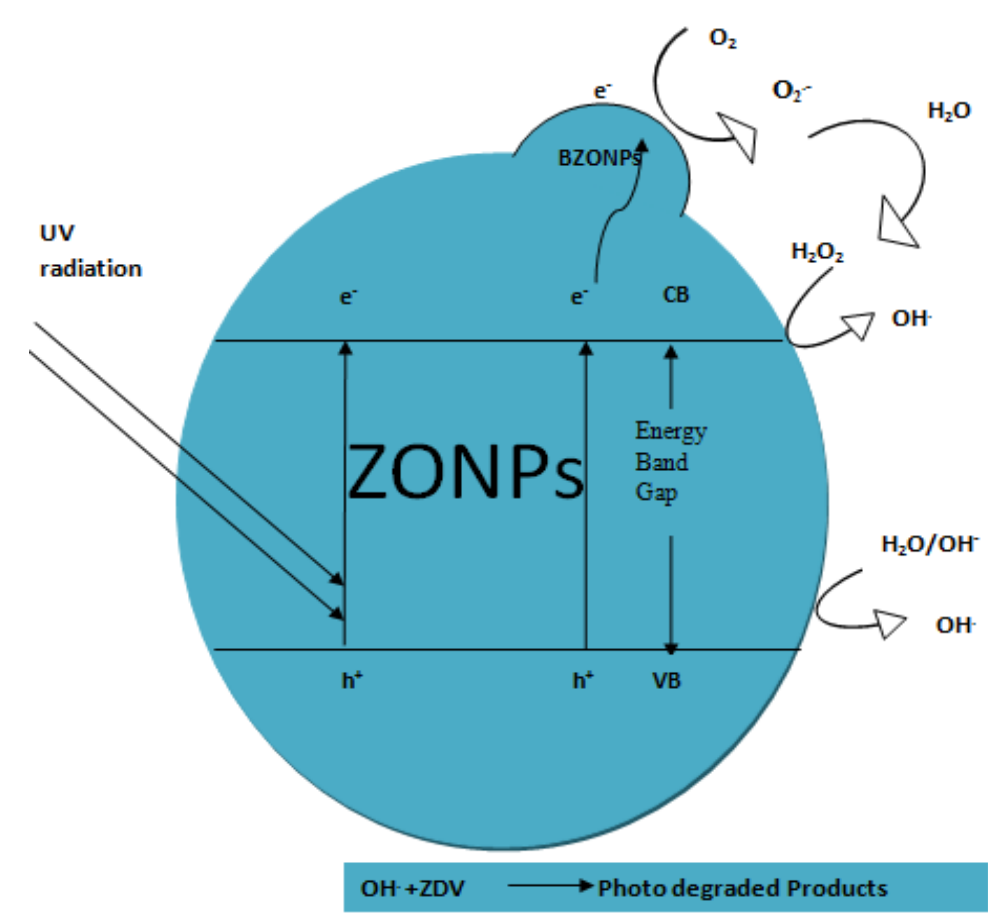

Fig. 12: Photocatalytic electron hole pair mechanism of ZDV with 5 \% (mole ratio) BZONPs

\section{CONCLUSION}

A suitable electron-hole pair mechanism is projected in agreement with results. The average grain size of $5 \%$ (mole ratio) BZONPs indicates that its surface area is increased. This concludes that the rate of photo-degradation of ZDV is faster using $5 \%$ (mole ratio) BZONPs. XRD patterns confirm that the synthesized ZONPs and 5\% (mole ratio) BZONPs are having wurzite structure. SEM images show that ZONPs and 5\% (mole ratio) BZONPs are uniform and homogeneous with closely aggregate oval in shape. EDX results concludes that there are only three elements present in $5 \%$ (mole ratio) BZONPs. There is an increase in the rate of photocatalytic degradation of ZDV by adding different quantities of photocatalyst from $0.05 \mathrm{~g} \mathrm{l}^{-1}$ to $0.1 \mathrm{~g} \mathrm{l}^{-1}$. The kinetic data indicates that $\mathrm{k}_{\mathrm{obs}}$ values are higher at $\mathrm{pH}=9$. There is an increase in the $\mathrm{k}_{\mathrm{obs}}$ values with increase in light intensities of UV lamp. Experimental results conclude that $5 \%$ (mole ratio) BZONPs is a very efficient photocatalyst for the photo-degradation of ZDV present in environmental waters.

\section{ACKNOWLEDGMENT}

We would like to place on record our sincere thanks to (a) Principal Scientist, Center for Material Characterization, National Chemical Laboratory, Pune for HR-MS (b) Shivaji University Kolhapur for XRD (c) National Institute of Technology, Surathkal for SEM and TEM of the samples.

\section{AUTHORS CONTRIBUTIONS}

All the authors have contributed equally

\section{CONFLICT OF INTERESTS}

The authors have declared no conflict of interest

\section{REFERENCES}

1. Pinillos JF, Rojas L, Gallardo C. Adsorption of erlotinib to multiwalled carbon nanotubes. Int J Pharm Pharm Sci 2016;8:399-403.

2. Kummerer K. Pharmaceuticals in the environment, Sources, Fate, Effects, and Risks. 2nd ed. Berlin: Springer Heidelberg; 2004.

3. Patgar MB, Meti MD, Nandibewoor ST, Chimatadar SA. Kinetics and mechanism of oxidation of an antiarrhythmic drug procainamide hydrochloride by $\mathrm{Mn}$ (VII) in an aqueous sulphuric acid medium: a stopped-flow technique. Int J Pharm Pharm Sci 2014;6:583-8.

4. Ternes TA, Meisenheimer M, McDowell D, Sacher F, Brauch HJ, Haist-Gulde B, et al. Removal of pharmaceuticals during drinking water treatment. Environ Sci Technol 2002;36:3855-63.

5. Yang $\mathrm{H}$, Cheng $\mathrm{H}$. Controlling nitrite level in drinking water by chlorination and chloramination. Sep Purif Technol 2007;26: 392-6.

6. Lu J, Zhang T, Ma J, Chen Z. Evaluation of disinfection byproducts formation during chlorination and chloramination of dissolved natural organic matter fractions isolated from a filtered river water. J Hazard Mater 2009;162:140-5.

7. Zhang J, Guan X. Ru (III) catalyzed permanganate oxidation of bisphenol A. Desalin Water Treat 2014;52:4592-601.

8. Lin T, Pan S, Chen W, Yu C. Effect of potassium permanganate pre-oxidation on fouling and pore size of ultrafiltration membrane for drinking water treatment. Desalin Water Treat 2012;50:254-63.

9. Zhang J, Zhang Y, Wang H, Guan X. Ru (III) catalyzed permanganate oxidation of aniline at environmentally relevant pH. J Environ Sci 2014;26:1395-402.

10. Zhang J, Sun B, Guan HX, Wang H, Bao LH, Huang YY. Ruthenium nanoparticles supported on $\mathrm{CeO}_{2}$ for catalytic permanganate oxidation of butylparaben. Environ Sci Technol 2013;47:13011-9.

11. Zhang J, Sun B, Xiong X, Gao N, Song W, Du E, et al. Removal of emerging pollutants by $\mathrm{Ru} / \mathrm{TiO}_{2}$-catalyzed permanganate oxidation. Water Res 2014;63:262-70.

12. Esplugas S, Gimenez J, Conteras S, Pascual E, Rodriguez M. Comparison of different advanced oxidation processes for phenol degradation. Water Res 2002;36:1034-42.

13. Padmanabhan PVA, Sreekumar KP, Thiyagarajan TK, Satpute RU, Bhanumurthy K, Sengupta $\mathrm{P}$, et al. Nano-crystalline titanium dioxide formed by reactive plasma synthesis. Vacuum 2006;80:11-2.

14. Gaya UI, Abdullah AHJ. Heterogeneous photocatalytic degradation of organic contaminants over titanium dioxide: a review of fundamentals, progress, and problems. J Photochem Photobiol C 2008;9:1-12.

15. Von Sonntag C. Degradation of aromatics by advanced oxidation processes in water remediation: some basic considerations. J Water Supply Res Tech 1996;45:84-91.

16. Hoigne J. Inter-calibration of $\mathrm{OH}$ radical sources and water quality parameters. Water Sci Technol 1997;35:1-8. 
17. Pera Titus M, Garcia Molina V, Banos MA, Gimenez J, Esplugas S. Degradation of chlorophenols by means of advanced oxidation processes: a general review. Appl Catal B: Environ 2004; 47:219-56.

18. Haque MM, Muneer M. TiO2-mediated photocatalytic degradation of a textile derivative of a textile derivative, bromothymol blue, in aqueous suspensions. Dyes Pigm 2007;75:443-8.

19. Sakthivel S, Neppolian B, Shankar MV, Arabindoo B, Palanichamy M, Murugesan V. Solar photocatalytic degradation of azo dye: comparison of photocatalytic efficiency of $\mathrm{ZnO}$ and $\mathrm{TiO}_{2}$. Sol Energy Mater Sol Cells 2003;77:65-82.

20. Khodja AA, Sheili T, Pihichowski JF, Boule P. Photocatalytic degradation of 2-phenylphenol on $\mathrm{TiO}_{2}$ and $\mathrm{ZnO}$ in aqueous suspensions. J Photochem Photobiol A 2001;141:231-9.

21. Manyasree D, Peddi KM, Ravikumar R. CuO nanoparticles: synthesis, characterization and their bactericidal efficacy. Int J Appl Pharm 2017;9:71-4.

22. Shao R, Sun L, Tang L, Chen Z. Preparation and characterization of magnetic core-shell $\mathrm{ZnFe}_{2} \mathrm{O}_{4} @ \mathrm{ZnO}$ nanoparticles and their application for the photodegradation of methylene blue. Chem Eng J 2013;217:185-91.

23. Misra M, Kapur P, Singla ML. Surface plasmon quenched of near band edge emission and enhanced visible photocatalytic activity of Au@ZnO core-shell nanostructure. Appl Catal B: Environ 2014;150:605-11.

24. Saleh R, Djaja NF. UV light photocatalytic degradation of organic dyes with Fe-doped $\mathrm{ZnO}$ nanoparticles. Superlattices Microstruct 2014;74:217-33.

25. Kumar K, Chitkara M, Sandhu IS, Mehta D, Kumar S. Photocatalytic, optical and magnetic properties of Fe-doped $\mathrm{ZnO}$ nanoparticles prepared by chemical route. J Alloys Compd 2014;588:681-9.

26. Dutta PK, Pehkonen So, Sharma VK, Ray AK. Photocatalytic oxidation of arsenic (III): evidence of hydroxylradicals. Environ Sci Technol 2005;39:1827-34.

27. Wang R, Xin JH, Yang Y, Liu H, Xu L, Hu J. The characteristics and photocatalytic activities of silver-doped $\mathrm{ZnO}$ nanocrystallites. Appl Surf Sci 2004;227:312-7.

28. Stiver G. The treatment of influenza with antiviral drugs. Can Med Assoc J 2003;168:49-56.

29. Panda S, Pattnaik S, Maharana L, Botta GB, Mohapatra P. Formulation and evaluation of zidovudine loaded olibanum resin microcapsules: exploring the use of natural resins as biodegradable polymeric materials for controlled release. Asian J Pharm Clin Res 2013;6:191-6.

30. Kummerer $K$. The presence of pharmaceuticals in the environment due to human use-present knowledge and future challenges. J Environ Manage 2009;90:2354-66.

31. Buchberger WW. Novel analytical procedures for screening of drug residues in water, wastewater, sediment, and sludge. Anal Chim Acta 2007;593:129-39.

32. Kumar P, Keshava Kumar PS, Kulkarni RM. Fate of zidovudine through water treatment with chlorine: a kinetic study. Int Res J Environ Sci 2014;3:50-5.

33. Fisch MA, Richman DD, Grieco MH, Gottlieb MS, Volberding PA, Laskin OL, et al. The efficacy of azidothymidine (AZT) in the treatment of patients with AIDS and AIDS-related complex. A double-blind placebo-controlled trial. N Engl J Med 1987;317:185-91.

34. Bukkitgar SD, Shetti NP, Kulkarni RM, Nandibewoor ST. Electro-sensing base for mefenamic acid on a $5 \%$ bariumdoped zinc oxide nanoparticle modified electrode and its analytical application. RSC Adv 2015;5:104891-9.

35. Water W, Te-Hua F, Liang-Wen J, Teen-Hang M, Yin-Shing Y. Surface morphology and liquid sensor sensitivity of bariumdoped ZnO thin film. J Sci Innov 2011;1:25-32.

36. Ozkan S, Kumthekar MW, Karakas G. Characterization and temperature-programmed studies over $\mathrm{Pd} / \mathrm{TiO}_{2}$ catalysts for NO reduction with methane. Catal Today 1998;40:3-14.

37. Lin HF, Liao SC, Hung SW, The dc thermal plasma synthesis of $\mathrm{ZnO}$ nanoparticles for visible-light photocatalyst. J Photochem Photobiol A 2005;174:82-7.

38. Taicheng A, Yang $\mathrm{H}$, Song $\mathrm{W}$, Li G, Luo H, William JC. Mechanistic considerations for the advanced oxidation treatment of fluoroquinolone pharmaceutical compounds using $\mathrm{TiO}_{2}$ heterogeneous catalysis. J Phy Chem A 2010;114:2569-75.

39. Sun J, Qiao L, Sun S, Wang G. Photocatalytic degradation of Orange $\mathrm{G}$ on nitrogen-doped $\mathrm{TiO}_{2}$ catalyst under visible light and sunlight irradiation. J Hazard Mater 2008;155:312-9.

40. Wang CC, Lee CK, Lyu MD, Juang LC. Photocatalytic degradation of C. I. Basic Violet using $\mathrm{TiO}_{2}$ catalysts supported by Y. Zeolite an investigation of the effects of operational parameters. Dyes Pigm 2008;76:312-9.

41. Peill NJ, Hoffmann MR. Mathematical model of a photocatalytic fiber-optic cable reactor for heterogeneous photocatalysis. Environ Sci Technol 1998;32:398-404.

42. Konstantinou IK, Albanis TA. $\mathrm{TiO}_{2}$-assisted photocatalytic degradation of azo dyes in aqueous solution: kinetic and mechanistic investigations a review. Appl Catal B: Environ 2004; 49:1-14.

43. Mahmoodi NM, Arami M, Limaee NY, Tabrizi NS. Kinetics of heterogeneous photocatalytic degradation of reactive dyes in an immobilized $\mathrm{TiO}_{2}$ photocatalytic reactor. J Colloid Interface Sci 2006;295:159-64.

44. Banerjee S, Gopal J, Muraleedharan P, Tyagi AK, Raj B. Physics and chemistry of photocatalytic titanium dioxide: visualization of bacterial activity using atomic force microscopy. Curr Sci 2006;90:1378-83.

45. Baruah S, Dutta J. Nanotechnology applications in pollution sensing and degradation in agriculture. Environ Chem Lett 2009;7:191-204.

46. Turchi CS, Ollis DF. Photocatalytic degradation of organic water contaminants: mechanisms involving hydroxyl radical attack. J Cat 1990;122:178-92

47. Ohko Y, Tatsuma T, Fujishima A. Characterization of $\mathrm{TiO}_{2}$ photocatalysis in the gas phase as a photoelectrochemical system: behavior of salt-modified system. J Phys Chem 2001;105:10016-21.

48. Kulkarni RM, Malladi RS, Hanagadakar MS, Doddamani MR Santhakumari B, Kulkarni SD. Ru-TiO2 semiconducting nanoparticles for the photo-catalytic degradation of bromothymol blue. J Mater Sci: Mater Electron 2016;27:13065-74. 